\title{
A Signal Sequence Mediates the Retrograde Transport of Proteins from the Axon Periphery to the Cell Body and Then into the Nucleus
}

\author{
Richard T. Ambron, ${ }^{1}$ Robert Schmied, ${ }^{1}$ Cheng-Chun Huang, ${ }^{2}$ and Marilyn Smedman ${ }^{2}$ \\ Departments of 'Anatomy and Cell Biology, and ${ }^{2}$ Otolaryngology, College of Physicians and Surgeons, Columbia \\ University, New York, New York 10032
}

The presynaptic terminal and axon of neurons can undergo structural changes in response to environmental signals. Since these changes require protein synthesis in the cell body, the needs of the periphery must somehow be communicated to the cell soma. To look for such a mechanism, we used artificial protein constructs with properties expected of a signal that is transported from the axon to the nucleus. One construct consisted of the nuclear import signal peptide (sp) of the SV40 large T antigen, coupled to human serum albumin (HSA) and rhodamine (r). When injected into the axoplasm of Aplysia californica neurons in vitro, the rHSA-sp was transported in the retrograde direction through the axon to the cell body and then into the nucleus. Little, if any, moved in the anterograde direction toward growth cones.

The retrograde movement of injected rHSA-sp was rapid ( $>25 \mathrm{~mm} / \mathrm{d}$ ) and depended upon intact microtubules. The sp portion of rHSA-sp provided access to both the retrograde transport system and the nuclear import apparatus. Thus, rHSA was not transported at all, but accumulated in organelles near the injection site. Also, rHSA-sp containing an sp with a Lys to Thr substitution, which is known to reduce nuclear import markedly, was transported only poorly. To look for endogenous molecules that use this system, we affinity-purified a rabbit polyclonal antibody to the signal sequence. The antibody recognized an $83 \mathrm{kDa}$ polypeptide on Western blots of Aplysia nervous tissue. These data indicate that Aplysia neurons contain the machinery to convey macromolecules from the axon periphery to the nucleus.

The functional interrelationship between the cell body, axon, and presynaptic terminal is complex given that all of the macromolecules required in the axon periphery must be manufactured in the cell soma (Ambron and Schwartz, 1979; Sherbany et al., 1979; Grafstein and Forman, 1980). The mechanisms that regulate the balance between synthesis in the cell body and protein utilization in the periphery are not understood. A further

Received Dec. 12, 1991; revised Feb. 19, 1992; accepted Feb. 24, 1992.

We thank Dr. Douglass Forbes for providing samples of the signal peptide constructs, Dr. Diane Garsetti for synthesizing the signal peptides, Drs. Robert N. Taub and Sindu Krishna for the confocal microscopy, and Drs. Michael Gershon and Eric Holtzman for their critical review of the manuscript. R.S. was supported by PHS Training Grant T32 AG 00189, and the work, by NIH Grant NS-22150.

Correspondence should be addressed to Richard Ambron, Ph.D., Anatomy and Cell Biology, 1204 Black Building, College of Physicians and Surgeons, Columbia University, New York, NY 10032.

Copyright (c) 1992 Society for Neuroscience 0270-6474/92/122813-06\$05.00/0 complication is the property of plasticity whereby the presynaptic terminal undergoes dramatic structural changes in response to environmental signals such as neurotransmitters (Bailey and Chen, 1983; Black et al., 1987), growth factors (Campenot, 1982; Davies, 1988), and other cues (Thompson ct al., 1982; Stevens and Landis, 1988; Nelson et al., 1989). Injury to peripheral axons can also elicit a major structural reorganization of the axonal arbor (Fawcett and Keynes, 1990). All of these changes, even though they occur in the periphery, require alterations in transcription in the cell body. This creates a dilemma: how are the needs of the axon and terminal communicated, often over great distances, to the nucleus? One way is to couple transcription to electrical activity (Sheng and Greenberg, 1990). Another is to transport macromolecular signals from the axon and synapse back to the cell body. Although the latter is an attractive idea that has long been postulated, especially to explain responses to peripheral nerve injury (Cragg, 1970; Walters et al., 1991), no retrogradely transported signal has ever been identified. The isolation and characterization of such signals would have implications for a variety of important neuronal functions, including nerve repair. Consequently, we have started to look for signal proteins using large neurons of Aplysia californica regenerating in vitro.

Aplysia neurons are excellent models for these experiments since they have large swellings on their axons and neurites that can be readily injected, thereby providing access to the axoplasmic compartment. Our approach was to use artificial constructs with properties expected of a signal that is retrogradely transported from the axon to the nucleus. Since proteins imported into the nucleus need a nuclear localization signal sequence (Newmeyer and Forbes, 1988; Goldfarb, 1989; Silver, 1991), we reasoned that such a sequence would be a minimal requirement. We therefore prepared a construct consisting of the nuclear import signal peptide (sp) of the SV-40 T antigen coupled to an inert protein, human serum albumin (HSA) (Goldfarb et al., 1986; Lanford et al., 1986). The construct was coupled to rhodamine (r) and was injected into terminal swellings in vitro. Injected rHSA-sp was rapidly transported in the retrograde direction to the cell body and then into the nucleus. Both retrograde transport and nuclear import required the intact sp, implying that these processes in Aplysia have receptors for the peptide and that endogenous proteins use this system. To search for such proteins, we prepared an affinity-purified antibody to the sp. The antibody recognized an $83 \mathrm{kDa}$ polypeptide on Western blots of Aplysia nervous tissue.

Some of these findings were published in preliminary form (Schmied and Ambron, 1991). 


\section{Materials and Methods}

Preparation of the nuclear import signal sequence construct for injection. The signal peptide (sp), $\mathrm{H}_{2} \mathrm{~N}$-Cys-Thr-Pro-Pro-Lys-Lys-Lys-Arg-LysVal-COOH (Lanford and Butel, 1984; Goldfarb et al., 1986), was prepared using a Waters 9050 solid-phase peptide synthesizer and was purified by HPLC using a C18 Delta Pack reverse-phase column. The peptide was coupled to HSA using $n$-maleimidobenzyl-hydroxysuccinimide (Pierce Chemicals, Rockford, IL) (Goldfarb et al., 1986). The increase in molecular weight seen after SDS-PAGE indicated that, on average, the HSA-sp contained 12-20 signal peptides, which is sufficient to mediate nuclear import (Dworetzky et al., 1988). HSA-sp was then reacted with TRITC (rhodamine) (Newmeyer et al., 1986). After coupling, unreacted rhodamine was removed by gel filtration or dialysis.

Growth and injection of neurons. Neurons were removed from the abdominal ganglion of juvenile Aplysia with a long segment of the original axon intact. Most of the cells used in this study were from the left upper quadrant of the ganglion. The cells were grown on plastic polylysine-coated dishes containing hemolymph:L15 (1:1) at $15^{\circ} \mathrm{C}$ (Schacher and Proshanski, 1983). Under these conditions, the cut end of the axon seals over to form a large terminal swelling.

Cells were injected during the third day of growth. One nanogram of

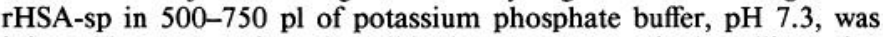
injected by pressure into the cell body, axon, or terminal swelling using micropipettes with $0.5 \mu \mathrm{m}$ tips fashioned with a Flaming-Brown electrode puller (Sutter Instrument Co., Novato, CA). After injection, cells were maintained at $15^{\circ} \mathrm{C}$ and fixed (Ambron et al., 1974). In experiments to determine the role of microtubules in transport, the cells were exposed to nocodazole ( $20 \mu \mathrm{M}$; Brabander et al., 1976) for $1 \mathrm{hr}$ before, and then throughout the period after injection. Cells were examined by epifluorescence on a Leitz microscope and by confocal microscopy using a Bio-Rad argon laser microscope.

Generation of an affinity-purified antibody to the signal peptide. $\mathrm{sp}$ was conjugated to maleimide-activated keyhole limpet hemocyanin (KLH; Pierce), and $25 \mu \mathrm{g}$ were injected into a rabbit. The animal was injected three more times at 6 week intervals. The IgG fraction from the serum was isolated using the ImmunoPure system as described by the manufacturer (Pierce). The IgG fraction was dialyzed and applied to a $2 \mathrm{ml}$ column of immobilized KLH. The run-through from that column was then added to a column of immobilized sp. The affinity columns were prepared according to Pierce Chemicals. Antibodies bound to the sp column were eluted with ImmunoPure elution buffer.

Identification of sp-containing proteins in the Aplysia nervous system. Ganglia and nerves from 10 animals were homogenized in $10 \mathrm{~mm}$ Trisbuffered saline ( $\mathrm{pH} 7.6$; TBS) containing leupeptin, pepstatin A, soybean trypsin inhibitor (each at $1 \mu \mathrm{g} / \mathrm{ml}$ ), $0.5 \mathrm{~mm}$ EGTA, and $0.6 \mathrm{~mm}$ phenylmethylsulfonyl fluoride, in a ground glass tissue grinder at $0^{\circ} \mathrm{C}$. The homogenate was centrifuged at $135,000 \times g$ for $20 \mathrm{~min}$ in a Beckman airfuge, and the supernatant $(930 \mu \mathrm{g})$ was dissolved in SDS sample buffer and distributed along a $5.5 \mathrm{~cm} 10 \%$ polyacrylamide gel. After SDSPAGE (Laemmli, 1970), the polypeptides were transferred to nitrocellulose paper. Strips were cut from the paper and sequentially incubated in $10 \mathrm{~mm}$ phosphate-buffered saline (pH 7.2), $0.2 \%$ glutaraldehyde in PBS, $0.05 \%$ Tween in TBS, and $1 \%$ gelatin in TBS. The glutaraldehyde step markedly improved the recognition of the polypeptides by the antibodies. To detect sp-containing polypeptides, the strips were exposed to $1 \%$ normal goat serum in TBS for $30 \mathrm{~min}$ and then to $20-50$ $\mu \mathrm{g} / \mathrm{ml}$ of the affinity-purified anti-sp antibody overnight at $4^{\circ} \mathrm{C}$. Bound

Figure 1. Transport of rHSA-sp to the nucleus after injection of the terminal swelling. A, Phase-contrast micrograph of an Aplysia neuron after $48 \mathrm{hr}$ in vitro. An extensive network of neurites has grown from the terminal swelling (arrow). This cell has a morphology typical of the neurons used in this study. In $B-E, 1$ shows the cell body, and 2 , the terminal. $C$, cytoplasm; $N$, nucleus. Approximately equal amounts of the various fluorescent constructs were injected. $B$, Bright-field micrograph of a neuron $24 \mathrm{hr}$ after injection of rHSA-sp into the terminal (arrow). The perimeter of the nucleus is shown by the broken line. $C$, Fluorescence micrograph of the cell in $B$ showing that the injected rHSAsp was transported to the nucleus; none remained at the site of injection. $D$. Fluorescence micrograph of a neuron $24 \mathrm{hr}$ after injection of the altered rHSA-sp. There is some staining of the cytoplasm and nucleus, but most of the fluorescence stayed at the injection site. $E$, Fluorescence micrograph of a neuron in the dish with the cell in $B$, but which was
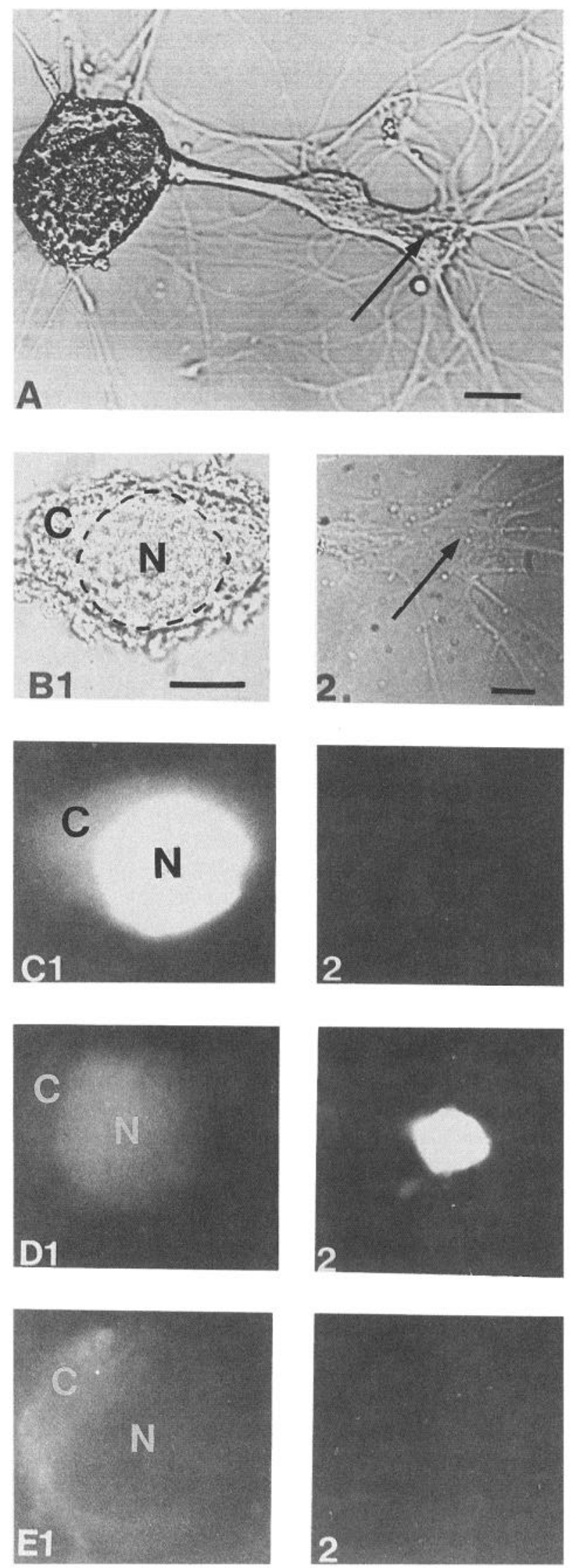

not injected, showing typical background autofluorescence. All the fluorescence photomicrographs in these experiments were taken at $60-75$ sec exposure. Scale bars: $A$ and $B I-E 1,50 \mu \mathrm{m} ; B 2-E 2,10 \mu \mathrm{m}$. 

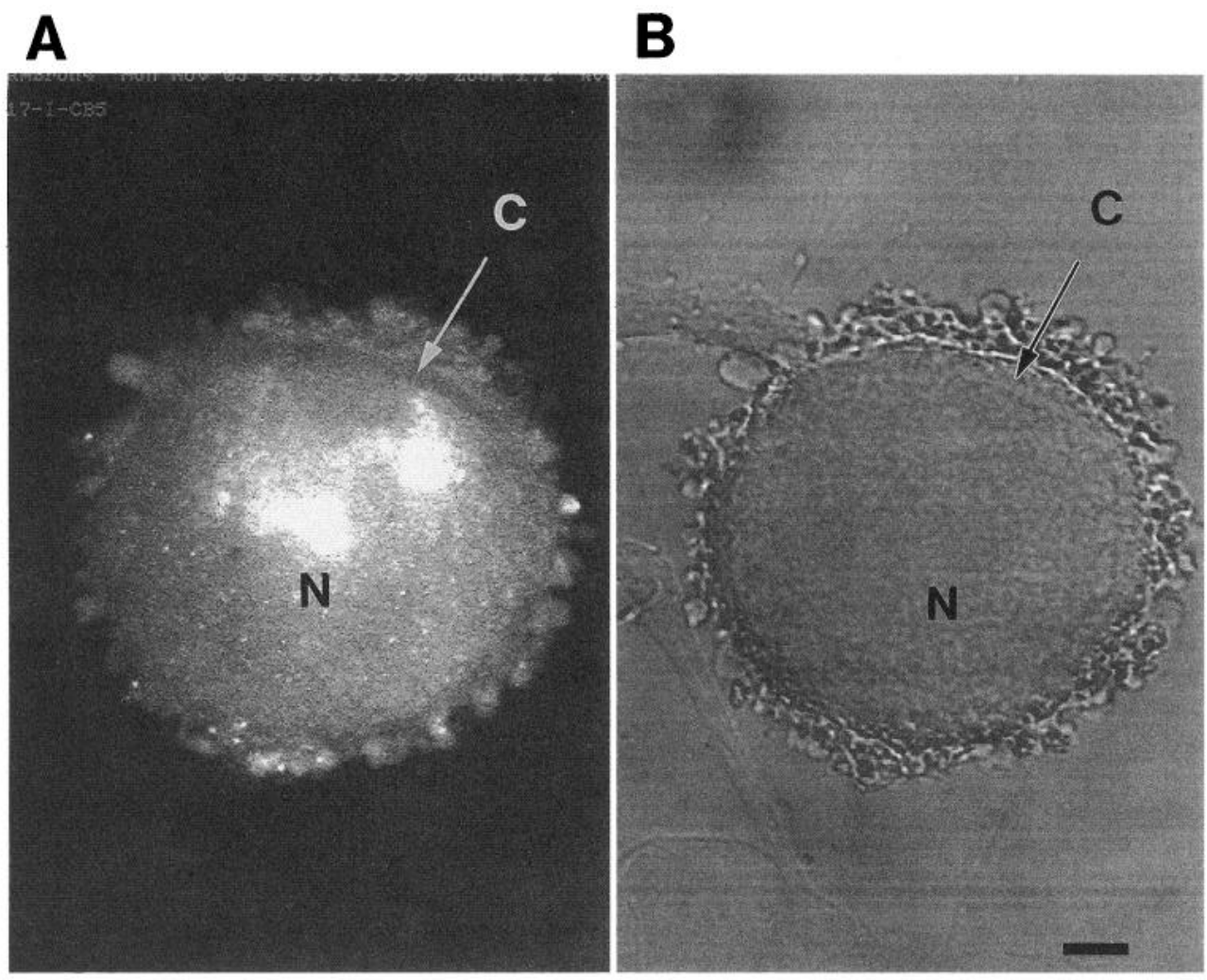

Figure 2. Confocal fluorescence microscopy of a cell body $24 \mathrm{hr}$ after injection of rHSA-sp into the terminal swelling: an optical section of the cell, taken at its maximum diameter, showing the nonuniform distribution of the rHSA-sp within the nucleus $(N)$. At this level the cytoplasm $(C)$ comprises only a narrow rim. $A$, fluorescence; $B$, phase contrast. Scale bar, $25 \mu \mathrm{m}$.

antibody was detected with affinity-purified goat anti-rabbit antibody conjugated to horseradish peroxidase $(200 \mathrm{ng} / \mathrm{ml})$ (Kierkegaard and Perry, Gaithersburg, MD). To show the specificity of the binding, the anti-sp antibody was incubated with $25 \mu \mathrm{g} / \mathrm{ml}$ of the signal peptide for $4 \mathrm{hr}$. The antibody-peptide mixture was then added to the nitrocellulose strip.

\section{Results}

When individual Aplysia neurons are removed from the ganglion and placed in culture, the severed axon seals over to form a terminal-like swelling from which neurites subsequently emerge (Fig. 1A). Similar swellings also appear along neurites as the cells in the culture grow older. In the experiments reported here, the terminal swellings were located $150-700 \mu \mathrm{m}$ from the cell body. Because the swellings are large, they can be microinjected; electron microscopy has shown that injected material has direct access to the axoplasm of the main axon and the growing neurites.

To probe for a system capable of transporting proteins from the axon to the nucleus, we coupled a nuclear import sp to HSA and made the construct fluorescent by conjugating it to TRITC (see Materials and Methods for details). We first tested the rHSAsp by injecting the construct into the cytoplasm of Aplysia neurons in vitro. Within $2 \mathrm{hr}$ after injection, most of the rHSA-sp was found in the nucleus (not shown). Having established that the nuclear import apparatus of Aplysia recognizes the sp, we then injected rHSA-sp directly into the terminal swelling of neurons in vitro. By $24 \mathrm{hr}$, essentially all of the construct in the 10 injected cells had been transported through the axon to the cell body and then into the nucleus (Fig. $1 B, C$ ). The nucleus of Aplysia neurons is large and often occupies most of the cell body. While fluorescence microscopy indicated that most of the construct had been imported into the nucleus, the distribution was more accurately determined by using confocal microscopy to section an injected cell optically (Fig. 2). The rHSA-sp was clearly within the nucleus, where it had a punctate pattern. The nuclear membrane was not labeled. The absence of fluorescence in the cytoplasm indicated that little or no rHSA-sp was directed to lysosomes or any other membranous compartment.

It is significant that transport occurred in all 30 experiments in which the rHSA-sp was injected into the axoplasm while, in the five experiments in which the construct was deposited directly onto the surface of the terminal, no staining was observed. This argues against the construct gaining access to the transport machinery by endocytosis. In addition, material that is endocytosed into Aplysia axons and transported to the cell body is found in lysosomes and Golgi-derived vesicles, and not the nucleus (Kistler and Schwartz, 1982). At first we thought that terminal swellings might be specialized for transport, but in three instances we succeeded in injecting the axon directly and found, as before, that the rHSA-sp was transported back to the nucleus.

One striking aspect of this transport was that the rHSA-sp moved through the axon almost exclusively in the retrograde direction; none was found in the neurites or at growth cones (Fig. 1C). This was more evident when we examined cells at much shorter times after injection. At $20 \mathrm{~min}$, for example, the bulk of the rHSA-sp had clearly moved toward the perikaryon and little, if any, in the anterograde direction toward the neurites (Fig. 3). Even after $3 \mathrm{hr}$, when the nucleus of most cells was already labeled, the distal processes were not stained much above background (Fig. 4A). Thus, the injected rHSA-sp had gained access to a mechanism that is capable of transporting proteins from the distal reaches of the axon to the nucleus.

The movement of the rHSA-sp through the axon is rapid, far more rapid than could be accounted for by diffusion (Koike and Nagata, 1979). The distribution of the construct along the axon 


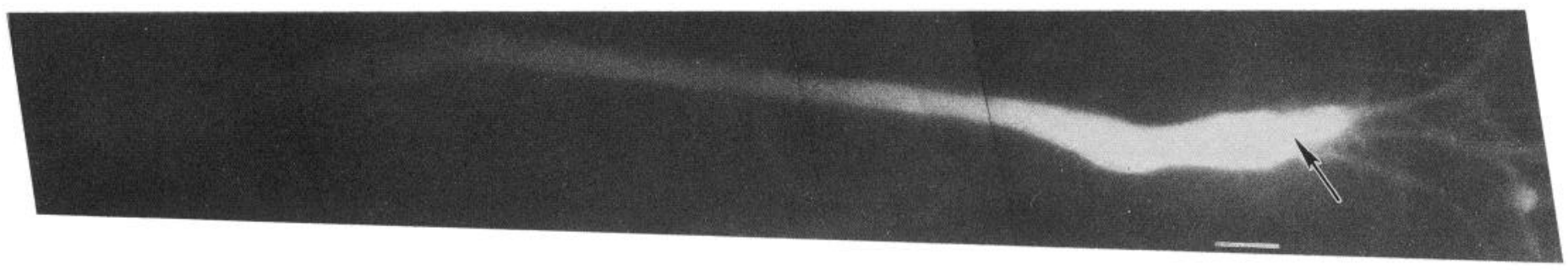

Figure 3. Fluorescence microscopy of a neuron $20 \mathrm{~min}$ after injection of rHSA-sp into the terminal swelling. The axon measured $650 \mu \mathrm{m}$ between the site of injection (arrow) and the cell body to the left (not shown). The bulk of the rHSA-sp moved toward the perikaryon, while little, if any, entered the neurites. Scale bar, $20 \mu \mathrm{m}$.

is also inconsistent with movement by diffusion (Koike and Nagata, 1979; Koike et al., 1989). Calculations based on observations at various times after injection determined that rHSAsp moves at a minimal rate of $25 \mathrm{~mm} / \mathrm{d}$, which is about half that of rapid anterograde transport in Aplysia (Ambron et al., 1974; Koike et al., 1989). These data are consistent with the idea that rHSA-sp utilizes the retrograde transport system that is known to exist in axons. If so, then its movement is dependent on microtubules and should be blocked by agents that disrupt microtubule function, such as nocodazole (Brabander et al., 1976). In confirmation of this idea, addition of the drug $(20 \mu \mathrm{M})$ to the bath $1 \mathrm{hr}$ prior to injection effectively prevented rHSAsp from leaving the injection site (Fig. $4 C$ ).

We considered it likely that rHSA-sp contains a specific signal that binds it to the retrograde transporter. To ascertain which part of the rHSA-sp is responsible for the binding, we examined the individual components of the construct. First, we injected rHSA and found, at both $3 \mathrm{hr}$ (Fig. $4 B$ ) and $24 \mathrm{hr}$ (not shown), that there was no staining of the cell body or axon; instead, the fluorescence became highly focalized in discrete structures near the injection site. This punctate pattern of staining was also observed with other rhodamine-conjugated polypeptides, including some endogenous proteins isolated from Aplysia axoplasm. Electron microscopy of terminals injected with colloidal gold coupled to the constructs showed that the rHSA is taken up into large lysosomal structures in the terminal (R. Schmied and R. T. Ambron, unpublished observations). Next, we injected unconjugated rhodamine and found that within $30 \mathrm{~min}$ the dye completely filled the growth cones, neurites, axon, and cell body except for the nucleus (not shown). This pattern was never seen after injection of protein constructs.

These results indicated that the sp was responsible for binding to the retrograde transport system. To confirm this deduction, we used rHSA coupled to an altered form of the sp in which threonine was substituted for one of the lysines; others have shown that this altered peptide is only about $15 \%$ as efficient in mediating entry into the nucleus (Goldfarb et al., 1986). When the altered rHSA-sp was injected, we found in all five cells that most of the construct remained in the terminal swelling for as long as $24 \mathrm{hr}$ and that both the cytoplasm and nucleus were only weakly stained (Fig. $1 D$ ). This stands in contrast to the behavior of the wild-type rHSA-sp, where the axoplasm was not stained and where most of the construct had already moved from the injection site by $3 \mathrm{hr}$ (Fig. $4 A$ ) and was gone by $24 \mathrm{hr}$ after injection (Fig. 1C). Thus, the diminished ability of the mutated rHSA-sp to gain entry to the nucleus was paralleled by its inefficiency in gaining access to the transport system, indicating that the $\mathrm{sp}$ is required for binding to both the retrograde transport system and the nuclear import apparatus. The altered construct uses the same transport mechanism as rHSA-sp since even its limited movement was blocked by nocodazole (not shown).

The fact that the Aplysia retrograde transport system recognizes sp implies that there are endogenous proteins with a similar amino acid sequence that utilize this system. Evidence for retrogradely transported protein in Aplysia has recently been reported (Walters et al., 1991). To look for such proteins, we generated a rabbit polyclonal antibody to the nuclear import signal sequence. Antibodies that recognized the sequence were isolated using an affinity column to which the peptide had been covalently coupled. We first used the affinity-purified antibody to probe the nervous system using immobilized proteins from the soluble and membrane fractions of isolated nerves and ganglia. A positive response was seen only among the soluble protein pool, which is consistent with the transport of soluble rHSA-sp. We then searched for individual polypeptides on Western blots and found that the antibody reacted strongly with an approximately $83 \mathrm{kDa}$ species (Fig. 5). Polypeptides of 75 , 110 , and $>200$ were also recognized, but these are not clearly discernible in the photograph. Antibody specificity was indicated by the fact that the binding to the $83 \mathrm{kDa}$ constituent was blocked by the sp.

\section{Discussion}

Structural changes in the axon and at presynaptic terminals require transcription in the cell body, yet the composition of those macromolecules destined for the axon and terminals can be dictated by events in the periphery. This interdependence implies that the needs of the periphery are somehow communicated to the cell soma. How does this communication occur? One way would be a mechanism whereby macromolecular signals from the axon or terminal are retrogradely transported to the soma, where they regulate the transcription of proteins destined for the periphery. We believe that by injecting our probes into the axoplasm of Aplysia neurons in vitro, we have gained access to such a signaling mechanism. This belief is based on the properties of the system. First, essentially all of the rHSAsp that moves from the injection site does so in the retrograde direction (Figs. 1C, 3, 4A). Over the course of more than 30 experiments we have injected wide-ranging amounts of proteinsp constructs into the axon. In only two instances did any construct enter the neurites, and these occurred when very large amounts were injected. Since movement of the rHSA-sp depended on an intact sp (see below), the sp binds to some element of the retrograde transporter and has little affinity for the anterograde transporter. This is consistent with the idea that mac- 

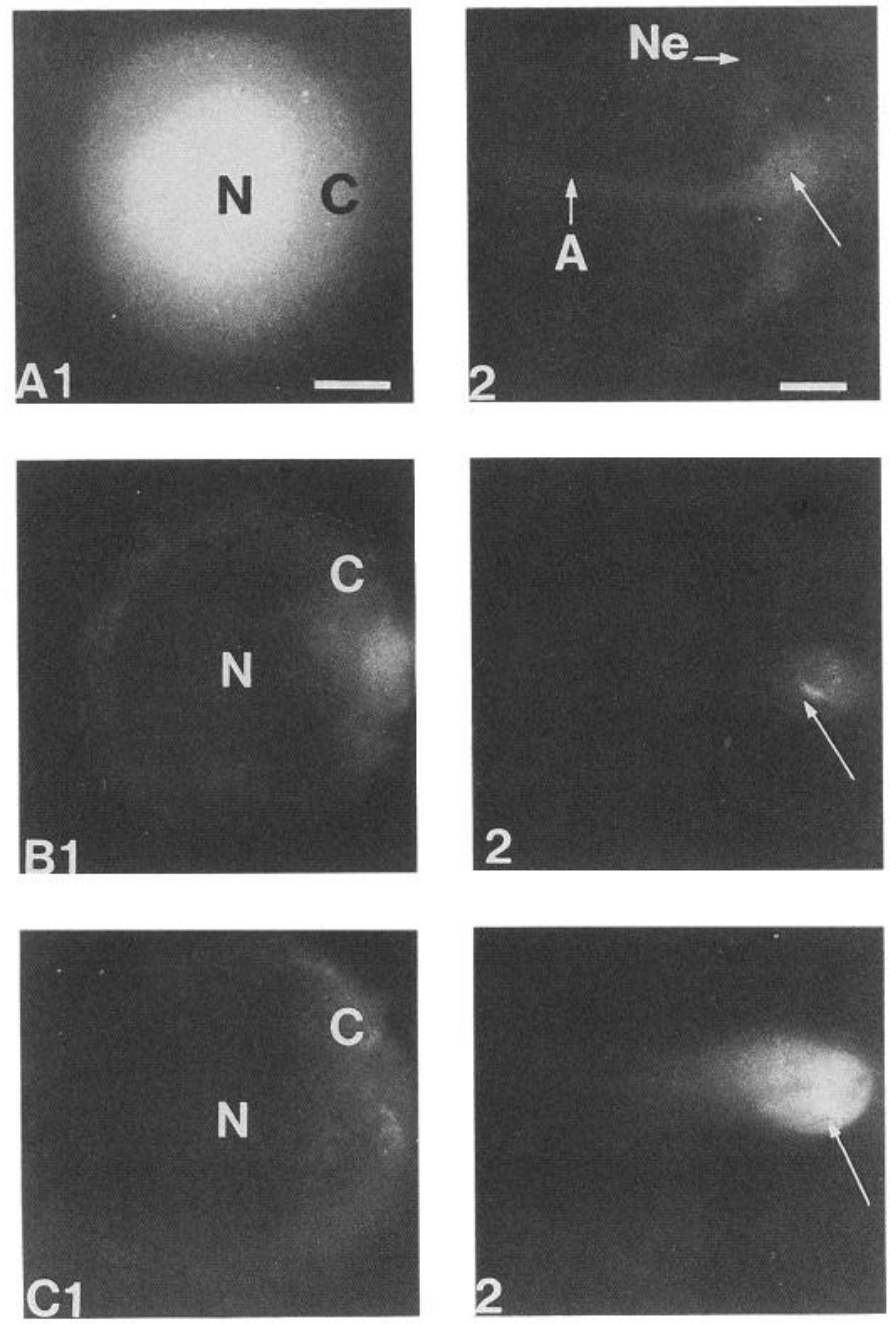

Figure 4. Fluorescence microscopy showing the distribution of rhodamine-coupled constructs $3 \mathrm{hr}$ after injection of the terminal. In $A-C$, 1 shows the cell body with cytoplasm $(C)$ and nucleus $(N)$, and 2 , the injection site (arrow) at the terminal swelling. Ne, neurites; $A$, axon. $A$, Injection of rHSA-sp. $B$, Injection of rHSA. Notice that neither the nucleus nor the cytoplasm is stained. Instead, intense fluorescence is seen in a punctate distribution near the injection site. The rHSA accumulates in structures, presumably lysosomes, throughout the terminal swelling. $C$. Injection of rHSA-sp into a cell that had been exposed to $20 \mu \mathrm{M}$ nocodazole for $1 \mathrm{hr}$ before injection; the fluorescence is confined to the injected terminal. The fluorescence photomicrographs were all taken at $65-80 \mathrm{sec}$ exposure. Scale bars: $A 1-C 1,50 \mu \mathrm{m} ; A 2-C 2,25 \mu \mathrm{m}$.

romolecules that use this mechanism are directed specifically to the cell soma. Second, the rapid retrograde movement of the construct along the axon and the dependence on microtubules, indicated by the experiments with nocodazole, strongly suggest that the construct moved via the retrograde transport machinery. How material that is injected into the axoplasm gains entry to this system is under investigation.

Third, both the transport of proteins to the cell body and their import into the nucleus depended on the intact sp; HSA without the sp remained at the injection site (Fig. $4 B$ ). In addition, when we used an altered signal sequence, one that permits nuclear import to only about $15 \%$ of normal (Lanford et al., 1986), retrograde transport of the construct was similarly reduced (Fig. $1 D$ ). Finally, the absence of a role for HSA was shown recently by attaching sp to the enzyme HRP. When HRP-sp was injected

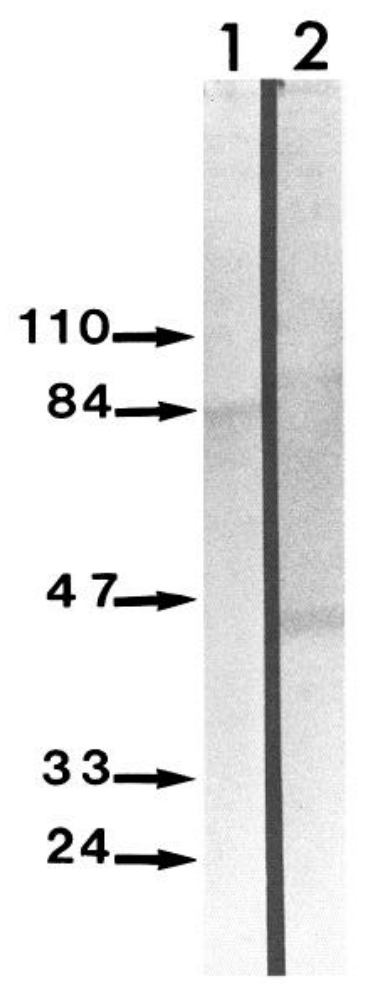

Figure 5. An affinity-purified antibody to the sp recognizes an $83 \mathrm{kDa}$ polypeptide on Western blots of Aplysia nervous tissue. The soluble fraction from Aplysia nervous tissue was separated by SDS-PAGE, and the polypeptides were transferred to nitrocellulose and probed with antibody (see Materials and Methods). Lane 1, Affinity-purified antibody. A prominent polypeptide of about $83 \mathrm{kDa}$ molecular weight is seen. Lane 2, Antibody in the presence of sp. The binding to the 83 $\mathrm{kDa}$ species is markedly reduced. Other stained bands appear, however. This occurs whenever a blot is exposed to the peptide. The explanation for this phenomenon is not known.

into the terminal, it was transported back to the nucleus (R. Schmied, D. A. Ambron, and R. T. Ambron, unpublished observations).

The discovery of this retrograde transport system implies that it is used for some purpose by neurons, but whether it conveys signals to the cell soma, as we propose, needs to be demonstrated. The existence of such signals has been postulated to explain the response of vertebrate neurons to axon injury (Cragg, 1970), and experiments showing that inhibitors of retrograde transport block the injury response support this idea (Singer et al., 1982). Invertebrate neurons also respond to injury. In Aplysia, for example, damage to the peripheral arbor of identified neurons alters the synthesis of certain glycoproteins (Goldberg and Ambron, 1986) and elicits reproducible changes in the electrophysiological properties of the cell body (Walters et al., 1991). The changes in electrical properties occurred even when injuryrelated action potentials were prevented from reaching the cell body, thereby eliminating electrical activity as the primary factor in eliciting the electrophysiological changes. In addition, the temporal relationship between the time of injury and the subsequent appearance of the electrophysiological events is consistent with the transport of a macromolecular signal from the injury site to the soma.

A first step toward proving that retrogradely transported signals exist in the axon would be to show that endogenous molecules use the transport/import pathway. We have evidence, 
albeit indirect, that this is the case. The recognition of the sp by both the transport and import machinery indicates that the receptor molecules are conserved and therefore important. This, in turn, implies that endogenous molecules are recognized by these receptors. If this is correct, then the endogenous proteins would have the same, or closely related, signal sequence. To test this idea, we generated an affinity-purified antibody to the signal sequence. The antibody detected several polypeptides, including a prominent $83 \mathrm{kDa}$ species, on Western blots of soluble extracts from Aplysia nervous tissue (Fig. 5). The antibody probably recognized the peptide sequence because the proteins were denatured by the SDS. Until the protein is activated, we would expect the signal sequence to be concealed so that the protein could be transported from the perikaryon to the terminals. Proteins with a hidden nuclear import signal are present in the cytoplasm of other cell types (Nagamine and Reich, 1985; Ghosh and Baltimore, 1990). Alternatively, the presence of the signal sequence in the Aplysia proteins might have nothing to do with transport or import. The resolution of this issue awaits the isolation of the $83 \mathrm{kDa}$ protein in its nondenatured form and its injection into the axon.

\section{References}

Ambron RT, Schwartz JH (1979) Regional aspects of neuronal glycoprotein and glycolipid synthesis. In: Complex carbohydrates of nervous tissue (Margolis RU, Margolis RK, eds), pp 269-289. New York: Plenum.

Ambron RT, Goldman JE, Schwartz JH (1974) Axonal transport of newly synthesized glycoproteins in a single identified neuron of $\mathrm{Aply}$ sia californica. J Cell Biol 61:665-675.

Bailey CH, Chen M (1983) Morphologic basis of long-term habituation and sensitization in Aplysia. Science 220:91-93.

Black IB, Adler JE, Dreyfus CF, Friedman WF (1987) Biochemistry of information storage in the nervous system. Science 236:1263-1268.

Brabander MJ, Van de Veire RML, Aerts FEM, Janssen AJ (1976) Effects of methyl [5-(2-thionylcarbonyl)-1H-benzimidol-2yl] carbamate, a new synthetic antitumoral drug interfering with microtubules, on mammalian cells cultured in vitro. Cancer Res 36:905-914.

Campenot RB (1982) Development of sympathetic neurons in compartmentalized cultures. Dev Biol 93:13-21.

Cragg BG (1970) What is the signal for chromatolysis? Brain Res 23: $1-21$.

Davies A (1988) The emerging generality of the neurotrophic hypothesis. Trends Neurosci 11:243-244.

Dworetzky SI, Lanford RE, Feldherr CM (1988) The effects of variations on the number and sequence of targeting signals on nuclear uptake. J Cell Biol 107:1279-1287.

Fawcett JW, Keynes RJ (1990) Peripheral nerve regeneration. Annu Rev Neurosci 13:43-60.

Ghosh S, Baltimore D (1990) Activation in vitro of NF-kB by phosphorylation of its inhibitor IkB. Nature 344:678-682.

Goldberg DE, Ambron RT (1986) Consequences of partial axotomy for production of neurotransmitter vesicles and routing of rapidly transported membrane glycoproteins in the axonal tree. $J$ Neurosci $6: 1712-1718$.

Goldfarb DS (1989) Nuclear transport. Curr Opin Cell Biol 1:441446.
Goldfarb DS, Gariepy L, Schoolnik JG, Kornberg RD (1986) Synthetic peptides as nuclear localization signals. Nature 322:641-644.

Grafstein B, Forman D (1980) Intracellular transport in neurons. Physiol Rev 60:1167-1283.

Kistler HB, Schwartz JH (1982) Retrograde axonal transport of a wheat germ agglutinin-horseradish peroxidase conjugate in Aplysia californica. Brain Res 244:343-346.

Koike H, Nagata Y (1979) Selective axonal transport in a single cholinergic axon of Aplysia: role of colchicine-resistant microtubules. J Physiol (Lond) 295:397-417.

Koike H, Matsumoto H, Umitsu Y (1989) Intra-axonal diffusion of $\left[{ }^{3} \mathrm{H}\right]$ acetylcholine and $\left[{ }^{3} \mathrm{H}\right]$ gammabutyric acid in a neuron of Aplysia. Neuroscience 32:539-555.

Laemmli VK (1970) Cleavage of structural proteins during the assembly of the head of bacteriorphage T4. Nature 277:680-685.

Lanford RE, Butel JS (1984) Construction and characterization of an SV 40 mutant defective in nuclear transport of $T$ antigen. Cell 37: 801-813.

Lanford RE, Kanda P, Kennedy RC (1986) Induction of nuclear transport with a synthetic peptide homologous to the SV40 $\mathrm{T}$ antigen transport signal. Cell 46:575-582.

Nagamine Y, Reich E (1985) Gene expression and cAMP. Proc Natl Acad Sci USA 82:4606-4610.

Nelson PG, Yu C, Fields RD, Neale EA (1989) Synaptic connections in vitro: modulation of number and efficacy by electrical activity. Science 244:585-587.

Newmeyer DD, Forbes DJ (1988) Nuclear import can be separated into distinct steps in vitro: nuclear pore binding and translocation. Cell 52:641-653.

Newmeyer DD, Finlay DR, Forbes DJ (1986) In vitro transport of a fluorescent nuclear protein and exclusion of non-nuclear proteins. $J$ Cell Biol 103:2091-2102.

Schacher S, Proshanski E (1983) Neurite regeneration by Aplysia neurons in dissociated cell culture: modulation by Aplysia hemolymph and the presence of the initial axonal segment. J Neurosci 3:24032413.

Schmied R, Ambron RT (1991) A signal sequence mediates the rapid retrograde transport of proteins from the axon into the nucleus. Soc Neurosci Abstr 17:563.

Sheng M, Greenberg ME (1990) The regulation and function of c-fos and other immediate early genes in the nervous system. Neuron 4: $477-485$.

Sherbany AA, Ambron RT, Schwartz JH (1979) Membrane glycolipids: regional synthesis and axonal transport in a single identified neuron of Aplysia californica. Science 203:78-81.

Silver P (1991) How proteins enter the nucleus. Cell 64:489-497.

Singer PA, Mehler S, Fernandez HL (1982) Blockade of retrograde axonal transport delays the onset of metabolic and morphologic changes induced by axotomy. $\mathrm{J}$ Neurosci 2:1299-1306.

Stevens LM, Landis SC (1988) Developmental interactions between sweat glands and the sympathetic neurons which innervate them: effects of delayed innervation on neurotransmitter plasticity and glial maturation. Dev Biol 130:703-720.

Thompson RF, Berger TW, Madden J (1983) Cellular processes of learning and memory in the mammalian central nervous system. Annu Rev Neurosci 6:447-491.

Walters ET, Alizadeh H, Castro GA (1991) Similar neuronal alterations induced by axonal injury and learning in Aplysia. Science 253: 797-799. 